\title{
Errata
}

\section{Cavitation in superfluid helium-4 at low temperature}

H. Lambaré ${ }^{1}$, P. Roche ${ }^{1}$, S. Balibar ${ }^{1}$, H.J. Maris ${ }^{2}$, O.A. Andreeva ${ }^{3}$, C. Guthmann ${ }^{1}$, K.O. Keshishev ${ }^{3}$, and E. Rolley ${ }^{1}$

1 Laboratoire de Physique Statistique de l'École Normale Supérieure, associé au CNRS et aux Universités Paris 6 et 7 , 24 rue Lhomond 75231, Paris Cedex 05, France

2 Department of Physics, Brown University, Providence, RI 02912, USA

3 Academy of Sciences of Russia, Institute for Physical Problems, Kosygin Street 2, Moscow 117334, Russia

Eur. Phys. J. B 2, 381-391 (1998)

In equation (16) and Table 1, the units for the energy barrier (K) should be replaced by $10^{-16}$ ergs, or the numerical values divided by 1.38 .

\section{Random polyelectrolytes and polyampholytes in solution}

I. Borukhov ${ }^{1}$, D. Andelman ${ }^{1}$, and H. Orland ${ }^{2}$

1 School of Physics and Astronomy, Raymond and Beverly Sackler Faculty of Exact Sciences Tel-Aviv University, Ramat-Aviv 69978, Tel-Aviv, Israel

e-mail: itamar@post.tau.ac.il, andelman@post.tau.ac.il

2 Service de Physique Théorique, CE-Saclay, 91191 Gif-sur-Yvette Cedex, France e-mail: orland@spht.saclay.cea.fr

Eur. Phys. J. B 5, 869-880 (1998)

Equation (42) should read:

$$
\mathrm{K}_{0}=\frac{\left[\mathrm{A}^{+}\right]\left[\mathrm{OH}^{-}\right]}{[\mathrm{AOH}]}
$$

